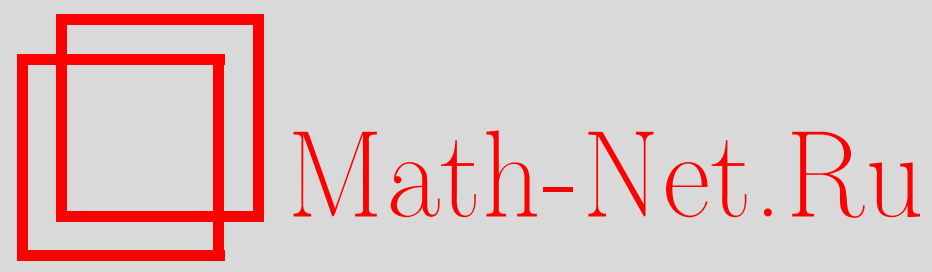

M. V. Nikolaev, Модифицированный алгоритм ГодриШоста для двумерной задачи дискретного логарифмирования, Матем. вопр. криптогр., 2020, том 11, выпуск 2, 125135

DOI: https://doi.org/10.4213/mvk326

Использование Общероссийского математического портала Math-Net.Ru подразумевает, что вы прочитали и согласны с пользовательским соглашением http://www.mathnet.ru/rus/agreement

Параметры загрузки:

IP: 52.90 .164 .192

26 апреля 2023 г., 17:56:00

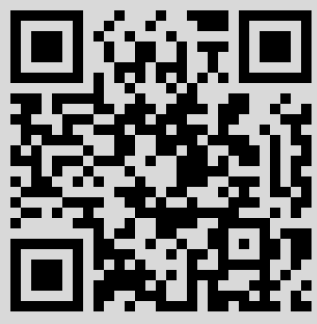




\title{
Modified Gaudry-Schost algorithm for the two-dimensional discrete logarithm problem
}

\author{
M. V. Nikolaev
}

Academy of Cryptography of the Russian Federation, Moscow

Получено 05.XI.2019

Abstract. We consider the two-dimensional discrete logarithm problem for the subgroup $G$ of a rational points group of an elliptic curve defined over a finite prime field that has an efficient automorphism of order 6 (for given $P_{1}, P_{2}, Q \in G, 0<N_{1}, N_{2}<\sqrt{|G|}$ we have to find $n_{1}, n_{2}$ such that $Q=n_{1} P_{1}+n_{2} P_{2},-N_{1} \leqslant n_{1} \leqslant N_{1},-N_{2} \leqslant n_{2} \leqslant N_{2}$ ). For this problem, modification of the Gaudry-Schost algorithm is suggested such that for any $\varepsilon>0$ there exists an algorithm with average complexity not exceeding $(1+\varepsilon) 0.847 \sqrt{N}+O_{\varepsilon}\left(N^{1 / 4}\right)$ group operations for $N=4 N_{1} N_{2}, N \rightarrow \infty$.

Keywords: Gaudry-Schost algorithm, two-dimensional discrete logarithm problem, efficient automorphism

\section{Модифицированный алгоритм Годри-Шоста для двумерной задачи дискретного логарифмирования}

\section{М. В. Николаев}

Академия криптографии Российской Федерации, Москва

Аннотация. Рассматривается двумерная задача дискретного логарифмирования для подгруппы $G$ группы точек эллиптической кривой над конечным простым полем, обладающей эффективным автоморфизмом порядка 6 (для заданных $P_{1}, P_{2}, Q \in G$, $0<N_{1}, N_{2}<\sqrt{|G|}$ требуется найти такие $n_{1}, n_{2}$, что $Q=$ $\left.n_{1} P_{1}+n_{2} P_{2}, \quad-N_{1} \leqslant n_{1} \leqslant N_{1}, \quad-N_{2} \leqslant n_{2} \leqslant N_{2}\right)$. Для этой задачи конструктивно доказывается, что для любого $\varepsilon>0$ существует модификация алгоритма Годри-Шоста, средняя трудоемкость которой не превосходит $(1+\varepsilon) 0.847 \sqrt{N}+O_{\varepsilon}\left(N^{1 / 4}\right)$ групповых операций при $N=4 N_{1} N_{2}, N \rightarrow \infty$.

Ключевые слова: алгоритм Годри-Шоста, двумерная задача дискретного логарифмирования, эффективный автоморфизм 


\section{Two-dimensional discrete logarithm problem}

Definition 1. Discrete logarithm problem.

Given: group $G=\langle P\rangle, Q \in G$.

Find: $n \in\{0, \ldots,|G|-1\}$ such that $Q=n P$.

Definition 2. Two-dimensional discrete logarithm problem.

Given: group $G$; $P_{1}, P_{2}, Q \in G, N_{1}, N_{2} \in \mathbb{N}, Q=n_{1} P_{1}+n_{2} P_{2}$ for some (unknown) $n_{1} \in\left\{-N_{1}, \ldots, N_{1}\right\}, n_{2} \in\left\{-N_{2}, \ldots, N_{2}\right\}$.

Find: $n_{1}^{\prime}, n_{2}^{\prime} \in \mathbb{Z}$ such that $Q=n_{1}^{\prime} P_{1}+n_{2}^{\prime} P_{2}$.

Two-dimensional discrete logarithm problem arises in a number of contexts, for example, in the computation of the group order of the Jacobian of curves [9], in the complexity analysis of solving the discrete logarithm problem for exponents of bounded height [2]. In general case, the Gaudry-Schost algorithm [9] is the most efficient algorithm for solving the two-dimensional discrete logarithm problem. The basic idea of the algorithm may be described as follows. First, we define the so-called "tame" and "wild" sets":

$$
\begin{gathered}
T=\left\{-N_{1}, \ldots, N_{1}\right\} \times\left\{-N_{2}, \ldots, N_{2}\right\} \\
W=\left\{-N_{1}+n_{1}, \ldots, N_{1}+n_{1}\right\} \times\left\{-N_{2}+n_{2}, \ldots, N_{2}+n_{2}\right\} .
\end{gathered}
$$

Then, we compute in parallel pseudo-random sequences

$$
\begin{gathered}
x_{i} P_{1}+y_{i} P_{2}, \quad\left(x_{i}, y_{i}\right) \in T, i=1,2, \ldots, \\
Q+z_{j} P_{1}+w_{j} P_{2}, \quad\left(n_{1}+z_{j}, n_{2}+w_{j}\right) \in W, j=1,2, \ldots,
\end{gathered}
$$

until we get two identical elements in them:

$$
x_{k} P_{1}+y_{k} P_{2}=Q+z_{l} P_{1}+w_{l} P_{2},
$$

then we can find $n_{1}^{\prime}=x_{k}-z_{l}, n_{2}^{\prime}=y_{k}-w_{l}$.

The average complexity of the Gaudry-Schost algorithm is determined using the following result of Galbraith and Holmes.

Theorem 1 ([6], Theorem 1). Suppose that the following conditions are satisfied.

\footnotetext{
${ }^{1}$ Despite the fact that the $n_{1}, n_{2}$ are unknown, we can choose elements from $W$, as it will be shown later.
} 
1) We assume that there are balls of $C$ different colours. The $j$-th ball sampled has color $c \in\{1, \ldots, C\}$ (independently of all previous ball colors) with probability $r_{j, c}$ and there exist limits

$$
p_{c}=\lim _{n \rightarrow \infty} n^{-1} \sum_{k=1}^{n} r_{j, c}, \quad c \in\{1, \ldots, C\},
$$

such that $p_{1} \geqslant p_{2} \geqslant \cdots \geqslant p_{C}>0$. Let $b_{n, c}=p_{c}-n^{-1} \sum_{k=1}^{n} r_{j, c}$. We assume that there is a constant $K$ such that $\left|b_{n, c}\right| \leqslant K / n$ for all $c$.

2) There are $N^{\prime} \in \mathbb{N}$ distinct urns. If the $j$-th ball has color $c$, then it is allocated into the urn $i$ independently of previous colours and urns selections with the probability $q_{c, i}\left(N^{\prime}\right)$. There exists $d>0$ such that for every $c=1, \ldots, C$ and $i=1, \ldots, N^{\prime}$

$$
0 \leqslant q_{c, i} \leqslant d / N^{\prime}
$$

There exist constants $\alpha, \mu>0$ such that

$$
\left|\left\{i \in\left\{1, \ldots, N^{\prime}\right\}: q_{1, i}, q_{2, i} \geqslant \mu / N^{\prime}\right\}\right| \geqslant \alpha N^{\prime} .
$$

Let $Z_{N^{\prime}}$ be the first time that there are two balls of different colours in the same urn. Then

$$
\mathbf{M}\left(Z_{N^{\prime}}\right)=\sqrt{\frac{\pi}{2 A_{N^{\prime}}}}+O\left({N^{\prime}}^{1 / 4}\right),
$$

where

$$
A_{N^{\prime}}=\sum_{c=1}^{C} p_{c}\left(\sum_{c^{\prime}=1, c \neq c^{\prime}}^{C} p_{c^{\prime}}\left(\sum_{i=1}^{N^{\prime}} q_{c, i} q_{c^{\prime}, i}\right)\right)
$$

and the constant in $O$ depends on $C, p_{c}, d, K, \alpha, \mu$, but does not depend on $N^{\prime}$ and $q_{c, i}\left(c \in\{1, \ldots, C\}, i \in\left\{1, \ldots, N^{\prime}\right\}\right)$.

The average complexity of the Gaudry-Schost algorithm was calculated by Galbraith and Ruprai in [7] and equals $(2.43+o(1)) \sqrt{N}$, where $N=$ $4 N_{1} N_{2}, o(1) \rightarrow 0, N_{1}, N_{2} \rightarrow \infty$. In the same paper an improved version of the algorithm was proposed with average complexity $(2.36+o(1)) \sqrt{N}$.

One can use the automorphism of group $G$ for which the orbit of any element of the group is calculated much faster than a group operation (such automorphism is called efficient) to speed up algorithm for solving the twodimensional problem. Let $G$ be a cyclic prime-order group and there exists 
an efficient automorphism $\varphi$ acting in the group $G$ as a multiplication by $\lambda \in\{1, \ldots,|G|-1\}$. Then, just as it is done for the classical discrete logarithm problem $[5,14]$, we can speed up the algorithm if we will search not for the same elements of the sequences (1) and (2), but for the same equivalence classes of these elements. Indeed, in this case, instead of equality (3), we have the equality

$$
\varphi^{s}\left(x_{k} P_{1}+y_{k} P_{2}\right)=Q+z_{l} P_{1}+w_{l} P_{2}
$$

for some $s$, whence

$$
Q=\left(\lambda^{s} x_{k}-z_{l}\right) P_{1}+\left(\lambda^{s} y_{k}-w_{l}\right) P_{2},
$$

i. e. $n_{1}^{\prime}=\lambda^{s} x_{k}-z_{l}, n_{2}^{\prime}=\lambda^{s} y_{k}-w_{l}$.

Efficient automorphisms are widely used in software implementations of cryptographic mechanisms based on elliptic curves $[3,4]$ in order to obtain speedup of scalar multiplications. The performance gains may be obtained by rewriting of $k P$ as $k_{1} P+k_{2} \varphi(P)$, where $\varphi$ is an automorphism of an elliptic curve, and $k_{1}, k_{2} \leqslant C_{\text {decomp }} \sqrt{|G|}$ for some constant $C_{\text {decomp }}$.

To compute $k P$ for a random $0<k<|G|$ (by GLV [10], GLS [11] methods) one of the following two approaches [1] is used.

1) The parameters $k_{1}, k_{2} \in_{R}[0, \sqrt{|G|}) \cap \mathbb{Z}$ are selected, and then point $k P=k_{1} P+k_{2} \varphi(P)$ is calculated, where $k \equiv k_{1}+\lambda k_{2} \bmod |G|$. This approach is called recomposition.

2) A random parameter $k$ is selected, and then corresponding $k_{1}, k_{2}$ are determined to calculate a point. This approach is called decomposition and is more resource intensive.

In case of using recomposition or decomposition, the problem of computing the discrete logarithm $k$ may be reduced to computing values of $k_{1}, k_{2}$ that satisfying

$$
k P=k_{1} P+k_{2} \varphi(P),
$$

i. e. to to the solution of the two-dimensional discrete logarithm problem with $P_{2}=\varphi\left(P_{1}\right)$.

There are several modifications of the Gaudry-Schost algorithm for elliptic curves with efficient automorphism.

1) In the case of subgroup of an elliptic curve $y^{2}=x^{3}+A x+B$ over a finite prime field with $p>3$ elements (it has an efficient automorphism of order 2) with $N_{1}=N_{2}$ there exists an algorithm with average 
complexity $(1+\varepsilon) 1.2533 \sqrt{N}+O_{\varepsilon}\left(N^{\frac{1}{4}}\right)$ group operations [13], where $N=4 N_{1} N_{2}$.

2) In the case of a subgroup of an elliptic curve $y^{2}=x^{3}+A x$ over a finite prime field with $p \equiv 1 \bmod 4$ elements, $P_{2}=\varphi\left(P_{1}\right)$ and $N_{1}=$ $N_{2}$, where $\varphi$ is an efficient automorphism of order 4 , there exists an algorithm with average complexity $(1+\varepsilon) 0.8862 \sqrt{N}+O_{\varepsilon}\left(N^{\frac{1}{4}}\right)$ group operations [13], where $N=4 N_{1} N_{2}$.

3) In the case of a subgroup of an elliptic curve $y^{2}=x^{3}+B$ over a finite prime field with $p \equiv 1 \bmod 3$ elements with $P_{2}=\varphi\left(P_{1}\right)$ and $N_{1}=$ $N_{2}$, where $\varphi$ is an efficient automorphism of order 6 , there exists an algorithm with average complexity $(1+\varepsilon) 0.8862 \sqrt{N}+O_{\varepsilon}\left(N^{\frac{1}{4}}\right)$ group operations [13], where $N=4 N_{1} N_{2}$.

In the latter case the automorphism of order 6 of an elliptic curve $y^{2}=x^{3}+B$ does not give a corresponding performance gain of $\sqrt{6}$ times. The purpose of this article is to obtain an optimized version of the GaudrySchost algorithm for this case. The main idea of modification of the Gaudry-Schost algorithm is to decompose the tame set into smaller ones and use non-uniform choice of elements from these subsets.

\section{Modified Gaudry-Schost algorithm}

Suppose that the prime-order- $q$ subgroup $G$ of an elliptic curve $E$ defined over $G F(p)$ by the equation $y^{2}=x^{3}+B$ with $p \equiv 1(\bmod 3), q^{2} \nmid \# E$ has an efficient automorphism $\varphi$ of order $6: \varphi(x, y)=(\beta x,-y)$, where $\beta \neq 1$ is a cube root of 1 modulo $p, \lambda$ is a root of the equation $\lambda^{2}-\lambda+1 \equiv 0$ $(\bmod q)$. For the point $P=(x, y)$ the equivalence class is represented by six elements:

$$
\left\{(x, y),(\beta x,-y),\left(\beta^{2} x, y\right),(x,-y),(\beta x, y),\left(\beta^{2} x,-y\right)\right\} .
$$

If $P_{2}=\varphi\left(P_{1}\right)$, then

$$
\varphi\left(a P_{1}+b P_{2}\right)=a\left(\lambda P_{1}\right)+b(\lambda-1) P_{1}=-b P_{1}+(a+b) P_{2},
$$

hence

$$
\begin{gathered}
\varphi^{2}\left(a P_{1}+b P_{2}\right)=-(a+b) P_{1}+a P_{2}, \\
\varphi^{3}\left(a P_{1}+b P_{2}\right)=-a P_{1}-b P_{2}, \\
\varphi^{4}\left(a P_{1}+b P_{2}\right)=b P_{1}-(a+b) P_{2}, \\
\varphi^{5}\left(a P_{1}+b P_{2}\right)=(a+b) P_{1}-a P_{2},
\end{gathered}
$$


i. e. the equivalence class of the point $a P_{1}+b P_{2}$ under the action of the group $\langle\varphi\rangle$ also includes the specified five points. Each such equivalence class corresponds to a set (class) of pairs

$$
\begin{aligned}
C(a, b)=\{(a, b),(-b, a+b),(- & (a+b), a), \\
& (-a,-b),(b,-(a+b)),(a+b,-a)\} .
\end{aligned}
$$

Theorem 2. Let $G$ be a prime-order-q subgroup of an elliptic curve $E$ defined over a finite prime field $G F(p)$ by the equation $y^{2}=x^{3}+B$ with $p \equiv$ $1(\bmod 3), q^{2} \nmid \# E$, let $\varphi$ be an automorphism of the group $G, \varphi(x, y)=$ $(\beta x,-y)$, where $\beta \neq 1$ is the cube root of $1 \operatorname{modulo} p$, let $\lambda$ be the root of the equation $\lambda^{2}-\lambda+1 \equiv 0(\bmod q)$ such that $\varphi(x, y)=\lambda(x, y)$. Then for any $\varepsilon>0$ there exists an algorithm for solving the two-dimensional discrete logarithm problem in $G$ with average complexity $(1+\varepsilon) 0.847 \sqrt{N}+O_{\varepsilon}\left(N^{\frac{1}{4}}\right)$ group operations (with $N_{1}=N_{2}, P_{2}=\varphi\left(P_{1}\right)$ and $\left(n_{1}, n_{2}\right)$ chosen uniformly at random), where $N=4 N_{1} N_{2}, N \rightarrow \infty$.

Proof. We define the "tame" set $T$ and the set $\widetilde{T}$ of representatives of the classes $T$ in the classical way:

$$
\begin{gathered}
T=\left\{C(a, b):-N_{1} \leqslant a \leqslant N_{1},-N_{1} \leqslant b \leqslant N_{1}\right\} . \\
\widetilde{T}=\left\{0, \ldots, N_{1}\right\} \times\left\{1, \ldots, N_{1}\right\} \cup\{(0,0)\} .
\end{gathered}
$$

We define the sets $T_{i}^{j}, j=0, \ldots, 5, i=1,2$, as follows (graphically represented on the Fig. 1):

$$
\begin{gathered}
T_{1}^{0}=\left\{(a ; b): 0 \leqslant a \leqslant N_{1}, 1 \leqslant b \leqslant N_{1}-a\right\}, \\
T_{2}^{0}=\left\{(a ; b): 0 \leqslant a \leqslant N_{1}, N_{1}-a+1 \leqslant b \leqslant N_{1}\right\}, \\
T_{i}^{j}=\varphi\left(T_{i}^{j-1}\right)=\left\{\varphi(a ; b):(a ; b) \in T_{i}^{j-1}\right\}, j=1, \ldots, 5, i=1,2 .
\end{gathered}
$$

Then

$$
T_{1}^{i} \cap T_{2}^{j}=\varnothing, \quad i, j=0, \ldots, 5, i \neq j, \quad \widetilde{T}=T_{1}^{0} \cup T_{2}^{0} .
$$




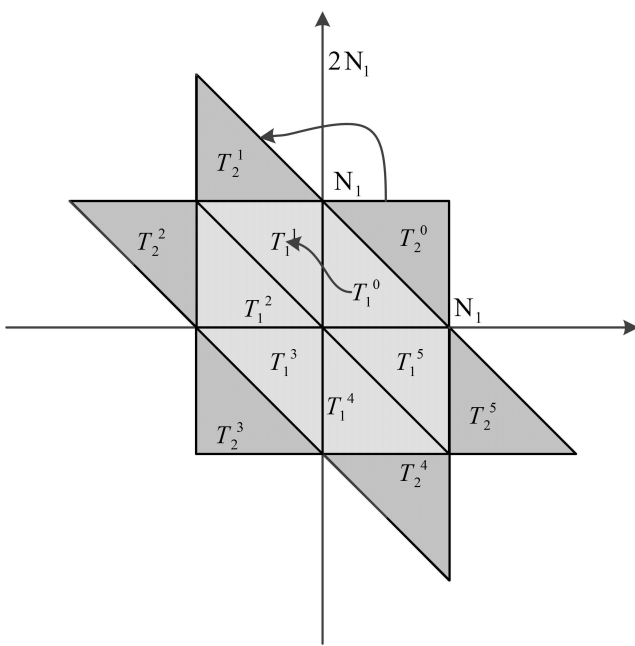

Figure 1: Structure of tame set

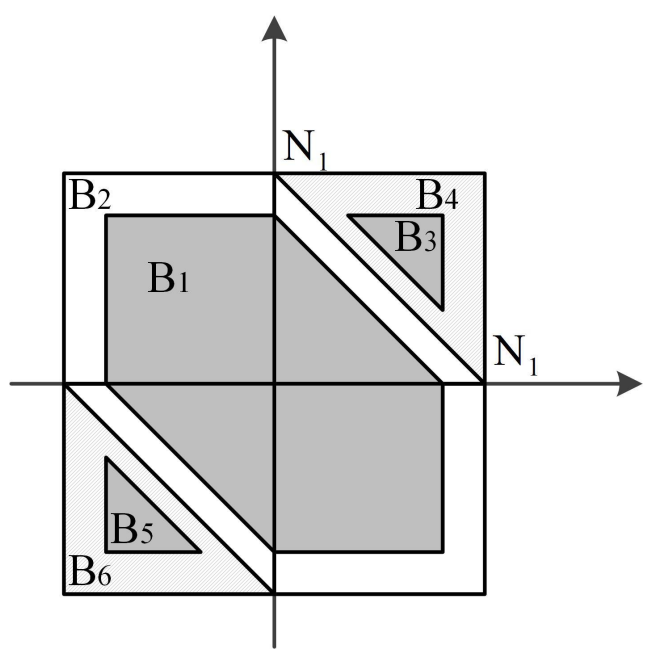

Figure 2: Values of $x, y$

Denote by $T_{0}$ the union of classes from the set $T$.

Define

$$
W_{k}=\left\{C\left(n_{1}+a, n_{2}+b\right):-\frac{k N_{1}}{2} \leqslant a \leqslant \frac{k N_{1}}{2},-\frac{k N_{1}}{2} \leqslant b \leqslant \frac{k N_{1}}{2}\right\}
$$

and

$$
\widetilde{W}_{k}=\left\{-\frac{k N_{1}}{2}+n_{1}, \ldots, \frac{k N_{1}}{2}+n_{1}\right\} \times\left\{-\frac{k N_{1}}{2}+n_{2}, \ldots, \frac{k N_{1}}{2}+n_{2}\right\} .
$$

We will compute tame and wild sequences as follows

$x_{i} P_{1}+y_{i} P_{2}$, where $\left\{\begin{array}{ll}\left(x_{i}, y_{i}\right) \in T_{1}^{0} & \text { with probability } p_{3}, \\ \left(x_{i}, y_{i}\right) \in T_{2}^{0} & \text { with probability } p_{4}=1-p_{3},\end{array} \quad i=1,2, \ldots\right.$,

$$
Q+z_{j} P_{1}+w_{j} P_{2},\left(z_{j}, \quad w_{j}\right) \in \widetilde{W}_{k}, j=1,2, \ldots
$$

Then average complexity measured in group operations does not exceed the total number $Z_{N^{\prime}}$ of values $\left(x_{i}, y_{i}\right)$ and $\left(z_{j}, w_{j}\right)$ chosen before the appearance of $\left(x_{l}, y_{l}\right)$ and $\left(z_{m}, w_{m}\right)$ such that $C\left(x_{l}, y_{l}\right)=C\left(z_{m}, w_{m}\right)$. Further we will estimate average number of steps of algorithm $Z_{N^{\prime}}$ as it was done in [13]. Under the conditions of the Galbraith-Holmes theorem, we have 
the following equalities:

$$
\begin{gathered}
p_{1}=p_{2}=\frac{1}{2}, \\
|T|=|\widetilde{T}|=\left|T_{1}^{0}\right|+\left|T_{2}^{0}\right|=\frac{N}{4}, \\
\left|T_{1}^{0}\right|=\left|T_{2}^{0}\right|=\frac{N}{8}, \\
\left|\widetilde{W}_{k}\right|=k^{2} \frac{N}{4} .
\end{gathered}
$$

However, balls of colour 1 (tame) will fall into urns from the sets $T_{1}^{0}$ and $T_{2}^{0}$ with probabilities $p_{3}$ and $p_{4}$, respectively $\left(p_{3}+p_{4}=1\right)$. Then

$$
q_{1, i}= \begin{cases}p_{3} \cdot \frac{8}{N}, & \text { if } i \in T_{1}^{0}, \\ p_{4} \cdot \frac{8}{N}, & \text { if } i \in T_{2}^{0}, \\ 0, & \text { otherwise }\end{cases}
$$

Since each class $C(a, b)$ contains no more than 6 elements, $T \cap W_{k}$ is divided into 6 disjoint subsets $U_{j}, j=1, \ldots, 6$, such that each class in $U_{j}$ gets exactly $j$ elements from $\widetilde{W}_{k}$, i. e.

$$
q_{2, i}=\frac{4 j}{N}, i \in U_{j}
$$

Besides,

$$
U=U_{1} \cup U_{2}=\left(U \cap T_{1}^{0}\right) \cup\left(U \cap T_{2}^{0}\right)
$$

We get:

$$
\begin{gathered}
A_{N^{\prime}}=\frac{1}{2}\left(\frac{8 p_{3}}{N} \frac{4}{k^{2} N}\left|U_{1}\right|+\frac{8 p_{4}}{N} \frac{4}{k^{2} N}\left|U_{2}\right|\right)=\frac{16}{k^{2} N^{2}}\left(p_{3}\left|U_{1}\right|+p_{4}\left|U_{2}\right|\right), \\
\mathbf{M}\left(Z_{N^{\prime}} \mid\left(n_{1}, n_{2}\right)\right) \\
=\sqrt{\frac{\pi}{2 A_{N^{\prime}}}}=\sqrt{\frac{\pi k^{2} N^{2}}{32\left(p_{3}\left|U_{1}\right|+p_{4}\left|U_{2}\right|\right)}} \\
=\sqrt{\frac{\pi}{32}} k \sqrt{\frac{1}{p_{3}\left|U_{1}\right|+p_{4}\left|U_{2}\right|}} .
\end{gathered}
$$

Following papers $[8,12]$, we set $n_{1}=x N_{1}, n_{2}=y N_{1},|x|,|y| \leqslant 1$. Let us estimate the cardinality of the set $U$ as function of the values of $x$ and $y$ (see Fig. 2). 
1) $\left(n_{1}, n_{2}\right) \in B_{1}=\left\{\left(x N_{1}, y N_{1}\right):-1+\frac{k}{2} \leqslant x \leqslant 1-\frac{k}{2}, \max (-1-x+\right.$ $\left.\left.k,-1+\frac{k}{2}\right) \leqslant y \leqslant \min \left(1-x-k, 1-\frac{k}{2}\right)\right\}$. Probability of the event $\left(n_{1}, n_{2}\right) \in B_{1}$ is equal $\frac{3}{4}-k+\frac{k^{2}}{4}$. In this case, the set $\widetilde{W}_{k}$ is completely contained in $\cup T_{1}^{j}, j=0, \ldots, 5$, i. e.

$$
\left|U_{1}\right|=\left|\widetilde{W}_{k}\right|
$$

2) $\left(n_{1}, n_{2}\right) \in B_{2}=\left\{\left(x N_{1}, y N_{1}\right):-1 \leqslant x \leqslant 1, \max (-1-x,-1) \leqslant y \leqslant\right.$ $\min (1-x, 1)\} \backslash B_{1}$. Probability of $\left(n_{1}, n_{2}\right) \in B_{2}$ is equal $k-\frac{k^{2}}{4}$. In this case we obtain the estimate

$$
\left|U_{1}\right| \geqslant \frac{\left|\widetilde{W}_{k}\right|}{4}
$$

3) $\left(n_{1}, n_{2}\right) \in B_{3}=\left\{\left(x N_{1}, y N_{1}\right): k+\frac{k}{2} \leqslant x \leqslant 1-\frac{k}{2}, 1-x+k \leqslant y \leqslant 1-\frac{k}{2}\right\}$. Probability of the event $\left(n_{1}, n_{2}\right) \in B_{3}$ is equal $\frac{k^{2}}{2}-\frac{k}{2}+\frac{1}{8}$. In this case, the set $\widetilde{W}_{k}$ is completely contained in $T_{0}$, i. e.

$$
\left|U_{2}\right|=\left|\widetilde{W}_{k}\right|
$$

4) $\left(n_{1}, n_{2}\right) \in B_{4}=\left\{\left(x N_{1}, y N_{1}\right): 0 \leqslant x \leqslant 1,1-x \leqslant y \leqslant 1\right\} \backslash B_{3}$. Probability of the event $\left(n_{1}, n_{2}\right) \in B_{4}$ is equal $-\frac{k^{2}}{2}+\frac{k}{2}$. In this case we obtain the estimate

$$
\left|U_{2}\right| \geqslant \frac{\left|\widetilde{W}_{k}\right|}{8}
$$

5) $\left(n_{1}, n_{2}\right) \in B_{5}=\left\{\left(x N_{1}, y N_{1}\right): k+\frac{k}{2} \leqslant x \leqslant 1-\frac{k}{2}, 1-x+k \leqslant y \leqslant 1-\frac{k}{2}\right\}$. Probability of the event $\left(n_{1}, n_{2}\right) \in B_{3}$ is equal $\frac{k^{2}}{2}-\frac{k}{2}+\frac{1}{8}$. In this case the set $\widetilde{W}_{k}$ is completely contained in $T_{0}$, i. e.

$$
\left|U_{2}\right|=\left|\widetilde{W}_{k}\right|
$$

6) $\left(n_{1}, n_{2}\right) \in B_{6}=\left\{\left(x N_{1}, y N_{1}\right): 0 \leqslant x \leqslant 1,1-x \leqslant y \leqslant 1\right\} \backslash B_{5}$. Probability of the event $\left(n_{1}, n_{2}\right) \in B_{4}$ is equal $-\frac{k^{2}}{2}+\frac{k}{2}$. In this case we obtain the estimate

$$
\left|U_{2}\right| \geqslant \frac{\left|\widetilde{W}_{k}\right|}{8} .
$$


Now we can estimate $\mathbf{M}\left(Z_{N^{\prime}}\right)$ :

$$
\begin{aligned}
& \mathbf{M}\left(Z_{N^{\prime}}\right) \\
& =\left(\frac{3}{4}-k+\frac{k^{2}}{4}\right) \mathbf{M}\left(Z_{N^{\prime}} \mid\left(n_{1}, n_{2}\right) \in B_{1}\right)+\left(k-\frac{k^{2}}{4}\right) \mathbf{M}\left(Z_{N^{\prime}} \mid\left(n_{1}, n_{2}\right) \in B_{2}\right) \\
& +2 \cdot\left(\frac{1}{4}-k+k^{2}\right) \mathbf{M}\left(Z_{N^{\prime}} \mid\left(n_{1}, n_{2}\right) \in B_{3}\right)+2 \cdot\left(k-k^{2}\right) \mathbf{M}\left(Z_{N^{\prime}} \mid\left(n_{1}, n_{2}\right) \in B_{4}\right) \\
& \quad \leqslant\left(\frac{3}{4}-k+\frac{k^{2}}{4}\right) \sqrt{\frac{\pi}{32}} k N \sqrt{\frac{4}{p_{3} k^{2} N}}+\left(k-\frac{k^{2}}{4}\right) \sqrt{\frac{\pi}{32}} k N \sqrt{\frac{16}{p_{3} k^{2} N}} \\
& +2 \cdot\left(\frac{1}{4}-k+k^{2}\right) \sqrt{\frac{\pi}{32}} k N \sqrt{\frac{4}{p_{4} k^{2} N}}+2 \cdot\left(k-k^{2}\right) \sqrt{\frac{\pi}{32}} k N \sqrt{\frac{16}{p_{4} k^{2} N}} \\
& \left.+\sqrt{\frac{\pi N}{32}}\left(\frac{3}{2}-2 k+\frac{k^{2}}{2}+4 k-k^{2}\right) \sqrt{\frac{1}{p_{3}}}+2 k+2 k^{2}+4 k-4 k^{2}\right) \sqrt{\frac{1}{p_{4}}} \\
& =\sqrt{\frac{\pi N}{32}}\left(\left(\frac{3}{2 \sqrt{p_{3}}}+\frac{1}{2 \sqrt{p_{4}}}\right)+2 k\left(\frac{1}{\sqrt{p_{3}}}+\frac{1}{\sqrt{p_{4}}}\right)-k^{2}\left(\frac{3}{4 \sqrt{p_{3}}}+\frac{2}{\sqrt{p_{4}}}\right)\right) .
\end{aligned}
$$

Let

$$
\varepsilon(k)=\left(2 k\left(\frac{1}{\sqrt{p_{3}}}+\frac{1}{\sqrt{p_{4}}}\right)-k^{2}\left(\frac{3}{4 \sqrt{p_{3}}}+\frac{2}{\sqrt{p_{4}}}\right)\right)\left(\frac{3}{2 \sqrt{p_{3}}}+\frac{1}{2 \sqrt{p_{4}}}\right)^{-1},
$$

then

$$
\mathbf{M}\left(Z_{N^{\prime}}\right) \leqslant(1+\varepsilon(k)) \frac{1}{8} \sqrt{\frac{\pi}{2}}\left(\frac{3}{\sqrt{p_{3}}}+\frac{1}{\sqrt{p_{4}}}\right) \sqrt{N} .
$$

The minimum of the function $f(x)=\left(\frac{3}{\sqrt{x}}+\frac{1}{\sqrt{1-x}}\right)$ at $(0 ; 1)$ is attained at $x_{0}=\frac{9}{10}-\frac{3 \sqrt[3]{3}}{10}+\frac{3^{2 / 3}}{10} \approx 0.67533$. Then for $p_{3}=0.67533, p_{4}=0.32467$

$$
\mathbf{M}\left(Z_{N^{\prime}}\right) \leqslant(1+\varepsilon) 0.847 \sqrt{N}+O_{\varepsilon}\left(N^{\frac{1}{4}}\right) .
$$

\section{Conclusion}

The modification of the Gaudry-Schost algorithm is proposed, which is the most effective for solving the two-dimensional discrete logarithm problem for an elliptic curve $y^{2}=x^{3}+B$. The performance gain was obtained by non-uniformly selection elements from various subsets of the tame set. 


\section{References}

[1] Aranha D. F., Fouque P., Gérard B., Kammerer J., Tibouchi M., Zapalowicz J.-C., "GLV/GLS decomposition, power analysis, and attacks on ECDSA signatures with singlebit nonce dias", ASIACRYPT 2014, Part I, Lect. Notes Comput. Sci., 8873, 2014, 262-281.

[2] Blackburn S.R., Scott S., "The discrete logarithm problem for exponents of bounded height", LMS J. Comput. and Math., 17 (2014), 148-156.

[3] Bos J. W., Costello C., Hisil H., Lauter K., "High-performance scalar multiplication using 8-dimensional GLV/GLS decomposition", CHES 2013, Lect. Notes Comput. Sci., 8086, 2013, 331-348.

[4] Costello C., Hisil H., Smith B., "Faster Compact Diffie-Hellman: Endomorphisms on the $x$-line", EUROCRYPT 2014, Lect. Notes Comput. Sci., 8441, 2014, 183-200.

[5] Duursma I. M., Gaudry P., Morain F., "Speeding up the discrete log computation on curves with automorphisms", Lect. Notes Comput. Sci., 1716, 1999, 103-121.

[6] Galbraith S. D., Holmes M., "A non-uniform birthday problem with applications to discrete logarithms", Discr. Appl. Math., 160:10-11 (2012), 1547-1560.

[7] Galbraith S. D., Ruprai R. S., "An improvement to the Gaudry-Schost algorithm for multidimensional discrete logarithm problems", IMACC 2009, Lect. Notes Comput. Sci., 5921, 2009, 368-382.

[8] Galbraith S. D., Ruprai R. S., "Using equivalence classes to accelerate solving the discrete logarithm problem in a short interval", PKC 2010, Lect. Notes Comput. Sci., 6056, 2010, 368-383.

[9] Gaudry P., Schost E., "A low-memory parallel version of Matsuo, Chao and Tsujii's algorithm", Lect. Notes Comput. Sci., 3076 (2004), 208-222.

[10] Gallant R., Lambert R., Vanstone S., "Faster point multiplication on elliptic curves with efficient endomorphisms", CRYPTO 2001, Lect. Notes Comput. Sci., 2139, 2001, 190-200.

[11] Galbraith S. D., Lin X., Scott M., "Endomorphisms for faster elliptic curve cryptography on a large class of curves", J. Cryptology, 24:3 (2011), 446-469.

[12] Liu W., Improved algorithms for the 2-dimensional discrete logarithm problem with equivalence classes, Master's thesis, Univ. of Auckland, 2010, http://www.math.auckland.ac.nz/ sgal018/Wei-Liu-MSc.pdf.

[13] Nikolaev M. V., "On the complexity of two-dimensional discrete logarithm problem in a finite cyclic group with efficient automorphism", Mathematicheskie Voprosy Kriptografi, 6:2 (2015), 45-57.

[14] Wiener M. J., Zuccherato R. J., "Faster attacks on elliptic curve cryptosystems", SAC 1998, Lect. Notes Comput. Sci., 1556, 1999, 190-200. 
